\title{
Finite element analysis of a nonlinear parabolic equation modeling epitaxial thin-film growth
}

\author{
Fengnan Liư ${ }^{1 *}$, Xiaopeng Zhao ${ }^{2}$ and Bo Liu ${ }^{1}$
}

\author{
"Correspondence: zxp032@yeah.net \\ 'College of Mathematics, Jilin \\ University, Changchun, 130012, \\ China \\ Full list of author information is \\ available at the end of the article
}

\begin{abstract}
In this paper, we consider a nonlinear model describing crystal surface growth. For the equation, the finite element method is presented and a nice error estimate is derived in the $L^{2}$ norm by means of a finite element biharmonic projection approximation.
\end{abstract}

\section{Introduction}

The finite element method is essentially a discretization method for the approximate solution of partial differential equations. It has the natural advantage of keeping the physical properties of the primitive problems. There are many papers that have already been published to study the finite element method for a fourth-order nonlinear parabolic equation (see [1-6]).

In this paper, we consider the finite element analysis for the following problem:

$$
\left\{\begin{array}{l}
\frac{\partial u}{\partial t}+\gamma u_{x x x x}-\left(\left|u_{x}\right|^{2} u_{x}-u_{x}\right)_{x}=0, \quad(x, t) \in(0, \pi) \times(0, T) \\
u_{x}(x, t)=u_{x x x}(x, t)=0, \quad x=0, \pi \\
u(x, 0)=u_{0}(x), \quad \text { in }(0, \pi),
\end{array}\right.
$$

where $\gamma$ is a positive constant.

Problem (1) arises in epitaxial growth of nanoscale thin films [7, 8], where $u(x, t)$ denotes the height from the surface of the film in epitaxial growth. The term $u_{x x x x}$ denotes the capillarity-driven surface diffusion, $u_{x x}$ denotes diffusion due to evaporationcondensation and $\left|u_{x}\right|^{2} u_{x}$ corresponds to the upward hopping of atoms. During the past years, many authors have paid much attention to problem (1), for example [7, 9-12].

Here, we give the existence and uniqueness of a global solution for problem (1) (see[7]).

Theorem 1.1 Suppose that $H_{E}^{2}(0, \pi)=\left\{u \in H^{2}(0, \pi): u_{x}(0)=u_{x}(\pi)=0\right\}$, and $u_{0} \in$ $H_{E}^{2}(0, \pi) \cap W^{1,4}(0, \pi)$, then there exists a unique global solution $u(x, t)$ for problem (1), such that

$$
u(x, t) \in C^{0}\left([0, T] ; L^{2}(0, \pi)\right) \cap L^{\infty}\left([0, T] ; H_{E}^{2}(0, \pi)\right) \cap L^{\infty}\left([0, T] ; W^{1,4}(0, \pi)\right) .
$$

The outline of this paper is as follows. In the next section, we establish a semi-discrete approximation and derive its error bound. In Section 3, the full-discrete approximation

\section{Springer}

○2014 Liu et al.; licensee Springer. This is an Open Access article distributed under the terms of the Creative Commons Attribution License (http://creativecommons.org/licenses/by/2.0), which permits unrestricted use, distribution, and reproduction in any medium, provided the original work is properly cited. 
for problem (1) is studied. In the last section, some numerical experiments which confirm our results are presented.

Throughout this paper, we denote the $L^{2}, L^{p}, L^{\infty}, H^{k}$ norms in $(0,1)$ simply by $\|\cdot\|,\|\cdot\|_{L^{p}}$, $|\cdot|_{\infty}$, and $\|\cdot\|_{k}$. Define the inner product of $L^{2}$ space as $(\cdot, \cdot)$, we have the space

$$
L^{p}(0, T ; X)=\left\{u(t):\|u\|_{L^{p}(X)}=\left(\int_{0}^{T}\|u(t)\|_{X}^{p} d t\right)^{\frac{1}{p}}<\infty\right\}, \quad 1 \leq p \leq \infty
$$

On the other hand, the letters $C, C^{\prime}$ denote generic constants independent of the finite element division size and not necessarily the same at different occurrences.

\section{Semi-discrete approximation}

Let $I_{h}: 0=x_{0}<x_{1}<\cdots<x_{N}=\pi$ be a finite element division for the interval $I=[0, \pi]$, $h_{i}=x_{i}-x_{i-1}, h=\max h_{i}$. Let $S_{h}^{(k)}$ be the piecewise polynomial spline space with the degree $k \geq 3$, and

$$
S_{h}^{(k)} \subset H_{E}^{2}(I)=\left\{u \in H^{2}(I): u_{x}(0)=u_{x}(\pi)=0\right\} .
$$

The weak formulation of problem (1) reads

$$
\left\{\begin{array}{l}
\left(u_{t}, v\right)+\gamma\left(D^{2} u, D^{2} v\right)+\left(|D u|^{2} D u-D u, D v\right)=0, \quad \forall v \in H_{E}^{2}(I), \\
u(x, 0)=u_{0}(x)
\end{array}\right.
$$

where $u_{t}=\frac{\partial u}{\partial t}, D u=\frac{\partial u}{\partial x}$. Based on (2), we define the semi-discrete finite element approximation to problem (1). Find $u_{h}(t):(0, T] \rightarrow S_{h}^{(k)}$ such that

$$
\left\{\begin{array}{l}
\left(u_{h, t}, v_{h}\right)+\gamma\left(D^{2} u_{h}, D^{2} v_{h}\right)+\left(\left|D u_{h}\right|^{2} D u_{h}-D u_{h}, D v_{h}\right)=0, \quad \forall v_{h} \in S_{h}^{(k)}, \\
\left(u_{h}(0)-u(0), v_{h}\right)=0, \quad \forall v_{h} \in S_{h}^{(k)}
\end{array}\right.
$$

It is clear that the conservation of mass for (3) holds as it does for the classical solution. Setting $v_{h}=1 \in S_{h}^{(k)}$ in (3), we get

$$
\int_{0}^{\pi} u_{h}(t, x) d x=\int_{0}^{\pi} u_{h}(0, x) d x, \quad 0 \leq t \leq T .
$$

Theorem 2.1 Let $u_{h}(0) \in H_{E}^{2}(I)$, then there exists a unique approximation solution $u_{h}(t) \in$ $S_{h}^{(k)}$ for problem (3), such that

$$
\left\|u_{h}(t)\right\|_{2} \leq C, \quad 0 \leq t \leq T
$$

where $C$ is a positive constant depending only on $\gamma$ and $\left\|u_{h}(0)\right\|_{2}$, independent of $h$.

Proof The equation of problem (3) is an ordinary differential equation and according to ODE theory, there exists a unique local solution to problem (3) in the interval $\left[0, t_{n}\right)$. If we have (4), then according to the extension theorem, we can also obtain the existence of unique global solution. So, we only need to prove (4). 
Setting $v_{h}=u_{h}$ in (3), we derive

$$
\begin{aligned}
& \frac{1}{2} \frac{d}{d t}\left\|u_{h}(t)\right\|^{2}+\gamma\left\|D^{2} u_{h}\right\|^{2}+\left(\left|D u_{h}\right|^{2} D u_{h}, D u_{h}\right) \\
& \quad=\left\|D u_{h}\right\|^{2}=-\left(u_{h}, D^{2} u_{h}\right) \leq \frac{\gamma}{2}\left\|D^{2} u_{h}\right\|^{2}+\frac{1}{2 \gamma}\left\|u_{h}\right\|^{2}
\end{aligned}
$$

Therefore

$$
\frac{d}{d t}\left\|u_{h}(t)\right\|^{2}+\gamma\left\|D^{2} u_{h}\right\|^{2} \leq \frac{1}{\gamma}\left\|u_{h}\right\|^{2} .
$$

Letting $\beta=\frac{1}{\gamma}$, we have

$$
\frac{d}{d t}\left(e^{-\beta t}\left\|u_{h}(t)\right\|^{2}\right) \leq 0
$$

Integrating (6) with respect to the time $t$, we get

$$
\left\|u_{h}(t)\right\|^{2} \leq e^{\beta t}\left\|u_{h}(0)\right\|^{2}, \quad 0 \leq t \leq T, \beta=\frac{1}{\gamma} .
$$

Setting $v_{h}=u_{h, t}$ in (3), we deduce that

$$
\left\|u_{h, t}\right\|^{2}+\gamma\left(D^{2} u_{h}, D^{2} u_{h, t}\right)+\left(\left|D u_{h}\right|^{2} D u_{h}-D u_{h}, D u_{h, t}\right)=0
$$

Let

$$
F_{h}(t)=\frac{\gamma}{2}\left\|D^{2} u_{h}\right\|^{2}+\frac{1}{4}\left\|D u_{h}\right\|_{L^{4}}^{4}-\frac{1}{2}\left\|D u_{h}\right\|^{2} .
$$

Differentiating $F_{h}(t)$ with respect to $t$, using (8), we get

$$
\begin{aligned}
\frac{d}{d t} F_{h}(t) & =\gamma \int_{\Omega} D^{2} u_{h} D^{2} u_{h, t} d x+\int_{\Omega}\left|D u_{h}\right|^{2} D u_{h} D u_{h, t} d x-\int_{\Omega} D u_{h} D u_{h, t} d x \\
& =-\left\|u_{h, t}\right\|^{2} \leq 0 .
\end{aligned}
$$

Therefore $F_{h}(t) \leq F_{h}(0)$, that is

$$
\begin{aligned}
\frac{\gamma}{2}\left\|D^{2} u_{h}\right\|^{2}+\frac{1}{4}\left\|D u_{h}\right\|_{L^{4}}^{4}+\frac{1}{2}\left\|D u_{h}(0)\right\|^{2} \\
\quad \leq \frac{\gamma}{2}\left\|D^{2} u_{h}(0)\right\|^{2}+\frac{1}{4}\left\|D u_{h}(0)\right\|_{L^{4}}^{4}+\frac{1}{2}\left\|D u_{h}\right\|^{2} \\
\quad \leq \frac{\gamma}{2}\left\|D^{2} u_{h}(0)\right\|^{2}+\frac{1}{4}\left\|D u_{h}(0)\right\|_{L^{4}}^{4}+\frac{\gamma}{4}\left\|D^{2} u_{h}\right\|^{2}+\frac{1}{4 \gamma}\left\|u_{h}\right\|^{2} \\
\quad \leq \frac{\gamma}{2}\left\|D^{2} u_{h}(0)\right\|^{2}+\frac{1}{4}\left\|D u_{h}(0)\right\|_{L^{4}}^{4}+\frac{\gamma}{4}\left\|D^{2} u_{h}\right\|^{2}+\frac{1}{4 \gamma} e^{\beta T}\left\|u_{h}(0)\right\|^{2} .
\end{aligned}
$$

Hence

$$
\left\|D^{2} u_{h}\right\|^{2} \leq C
$$


We also have

$$
\left\|D u_{h}\right\|^{2}=-\left(u_{h}, D^{2} u_{h}\right) \leq \frac{1}{2}\left\|u_{h}\right\|^{2}+\frac{1}{2}\left\|D^{2} u_{h}\right\|^{2} .
$$

By (7), (10), and (11), we complete the proof of Theorem 2.1.

Remark 2.1 By the above argument, we can obtain a better result. Let $C_{1}^{\prime}=\min \left\{\frac{\gamma}{4}, \frac{1}{4}\right\}$, $C_{2}^{\prime}=\max \left\{\frac{\gamma}{2}, \frac{1}{4}, \frac{1}{4 \gamma} e^{\beta T}\right\}$. It then follows from (9) that

$$
C_{1}^{\prime}\left(\left\|D^{2} u_{h}\right\|^{2}+\left\|D u_{h}\right\|_{L^{4}}^{4}\right) \leq C_{2}^{\prime}\left(\left\|D^{2} u_{h}(0)\right\|^{2}+\left\|D u_{h}(0)\right\|_{L^{4}}^{4}+\left\|u_{h}(0)\right\|^{2}\right) .
$$

Furthermore, we have

$$
\left\|u_{h}(t)\right\|_{2}^{2}+\left\|D u_{h}(t)\right\|_{L^{4}}^{4} \leq C_{1}\left(\left\|u_{h}(0)\right\|_{2}^{2}+\left\|D u_{h}(0)\right\|_{L^{4}}^{4}\right)
$$

where $C_{1}$ is a positive constant dependent only on $\gamma$, independent of $u_{h}(0)$ and $h$.

In order to consider the error estimate, we first introduce a finite element approximation projection for a steady-state problem. Let $u, v \in H_{E}^{2}(I)$, define $a(u, v) \equiv \gamma\left(D^{2} u, D^{2} v\right)+(u, v)$, define the biharmonic projection $R_{h}: u \rightarrow R_{h} u \in S_{h}^{(k)}$ such that

$$
a\left(u-R_{h} u, v_{h}\right) \equiv \gamma\left(D^{2}\left(u-R_{h} u\right), D^{2} v_{h}\right)+\left(u-R_{h} u, v_{h}\right)=0, \quad \forall v_{h} \in S_{h}^{(k)} .
$$

It then follows (12) that

$$
\|u\|_{2}^{2} \leq c_{0} a(u, u), \quad \forall u \in H_{E}^{2}(I)
$$

where $c_{0}$ is a positive constant depends only on $\gamma$ and $\mu$. Hence, $a(u, v)$ is a symmetrical positive determined bilinear form, and there exists a unique solution $u_{h} \in S_{h}^{(k)}$ for problem (12).

Based on the standard finite element method for a biharmonic equation (see [13]), we have

$$
\left\|u-R_{h} u\right\|+h\left\|u-R_{h} u\right\|_{1}+h^{2}\left\|u-R_{h} u\right\|_{2} \leq C h^{r+1}\|u\|_{r+1}, \quad 2 \leq r \leq k .
$$

Now, we consider the error estimate for the semi-discrete finite element solution. Let $u$ be the solution of (2), and $u_{h}$ be the solution of (3). Denote $\eta(t)=u-R_{h} u$ and $\theta(t)=$ $R_{h} u-u_{h}$, then

$$
u-u_{h}=u-R_{h} u+R_{h} u-u_{h}=\eta(t)+\theta(t) .
$$

Combining (2) and (4) gives

$$
\begin{aligned}
& \left(u_{t}-u_{h, t}, v_{h}\right)+\gamma\left(D^{2} u-D^{2} u_{h}, D^{2} v_{h}\right)+\left(|D u|^{2} D u-\left|D u_{h}\right|^{2} D u_{h}, D v_{h}\right) \\
& -\left(D u-D u_{h}, D v_{h}\right)=0
\end{aligned}
$$


that is

$$
\begin{aligned}
& \left(\eta_{t}+\theta_{t}, v_{h}\right)+\gamma\left(D^{2} \eta+D^{2} \theta, D^{2} v_{h}\right)+\left(|D u|^{2} D u-\left|D u_{h}\right|^{2} D u_{h}, D v_{h}\right) \\
& -\left(D \eta+D \theta, D v_{h}\right)=0 .
\end{aligned}
$$

It then follows from (12) and (16) that

$$
\begin{aligned}
& \left(\theta_{t}, v_{h}\right)+\gamma\left(D^{2} \theta, D^{2} v_{h}\right) \\
& \quad=\left(\eta-\eta_{t}, v_{h}\right)-\left(|D u|^{2} D u-\left|D u_{h}\right|^{2} D u_{h}, D v_{h}\right)+\left(D \theta, D v_{h}\right)
\end{aligned}
$$

Lemma 2.1 Let $u$ be the solution of $(2), u_{h}$ be the solution of $(3), u \in L^{\infty}\left(0, T ; H^{2}(I)\right)$. Then there exists a constant $C=C\left(u, u_{h}(0), \gamma\right)$ such that

$$
-\left(|D u|^{2} D u-\left|D u_{h}\right|^{2} D u_{h}, D \theta\right) \leq C\left(\|\theta\|^{2}+\|\eta\|^{2}\right)+\frac{\gamma}{4}\left\|D^{2} \theta\right\|^{2} .
$$

Proof First of all, we give some estimates which will be used in this proof. It follows from Theorem 2.1, (12), and (13) that

$$
\left\|u-u_{h}\right\|_{1} \leq\|u\|_{1}+C \leq C,
$$

and

$$
\|\eta\|_{2}=\left\|u-R_{h} u\right\|_{2} \leq\|u\|_{2}+C\|u\|_{2} \leq C \text {. }
$$

We notice that

$$
|D u|^{2} D u-\left|D u_{h}\right|^{2} D u_{h}=\left[(D u)^{2}+D u D u_{h}+\left(D u_{h}\right)^{2}\right]\left(D u-D u_{h}\right) .
$$

By Sobolev's embedding theorem, we have $H^{2}(I) \hookrightarrow W^{1, \infty}(I)$. Hence

$$
|D u|_{\infty} \leq C^{\prime}, \quad\left|D u_{h}\right|_{\infty} \leq C^{\prime}, \quad|D \theta|_{\infty} \leq C^{\prime}\|\theta\|_{2} .
$$

Thus, using the method of integration by parts, we get

$$
\begin{aligned}
&-\left(|D u|^{2} D u-\left|D u_{h}\right|^{2} D u_{h}, D \theta\right) \\
&=-\left(\left[(D u)^{2}+D u D u_{h}+\left(D u_{h}\right)^{2}\right]\left(D u-D u_{h}\right), D \theta\right) \\
&=\left(\left[(D u)^{2}+D u D u_{h}+\left(D u_{h}\right)^{2}\right](\theta+\eta), D^{2} \theta\right) \\
&+\left(\theta+\eta, D \theta\left[2 D u D^{2} u+D u D^{2} u_{h}+D^{2} u D u_{h}+2 D u_{h} D^{2} u_{h}\right]\right) \\
& \leq\|\theta+\eta\|\left\|D^{2} \theta\right\|+|D \theta|_{\infty} \\
& \times\|\theta+\eta\|\left(2|D u|_{\infty}\left\|D^{2} u\right\|+|D u|_{\infty}\left\|D^{2} u_{h}\right\|+\left|D u_{h}\right|_{\infty}\left\|D^{2} u\right\|+2\left|D u_{h}\right|_{\infty}\left\|D^{2} u_{h}\right\|\right) \\
& \leq\|\theta+\eta\|\left\|D^{2} \theta\right\|+C^{\prime}\|\theta\|_{2}\|\theta+\eta\| \\
& \leq C\left(\|\theta\|^{2}+\|\eta\|^{2}\right)+\varepsilon\left\|D^{2} \theta\right\|^{2} .
\end{aligned}
$$

Then the proof of Lemma 2.1 is completed. 
Remark 2.2 We use the integration by parts for the term $-\left(|D u|^{2} D u-\left|D u_{h}\right|^{2} D u_{h}, D \theta\right)$ in the proof of Lemma 2.1. Then a better convergency is obtained.

Theorem 2.2 Let $u$ be the solution of (2), $u_{h}$ be the solution of (3), $u(0) \in H^{k+1}(I), u_{t} \in$ $L^{2}\left(0, T ; H^{k+1}(I)\right)$, and the initial value satisfies

$$
\left\|u(0)-u_{h}(0)\right\| \leq C h^{k+1}\|u(0)\|_{k+1} .
$$

Then we have the following error estimate:

$$
\left\|u(t)-u_{h}(t)\right\| \leq C^{\prime} h^{k+1}\left(\|u(0)\|_{k+1}^{2}+\int_{0}^{T}\left\|u_{t}(\tau)\right\|_{k+1}^{2} d \tau\right)^{\frac{1}{2}}, \quad 0 \leq t \leq T,
$$

where the constant $C^{\prime}=C^{\prime}\left(u, u_{h}(0), \gamma\right)$.

Proof By (14)-(15) and Lemma 2.1, we only need to estimate $\theta(t)$. Setting $v_{h}=\theta$ in (17), using Cauchy's inequality, we immediately conclude that

$$
\begin{aligned}
\frac{1}{2} & \frac{d}{d t}\|\theta\|^{2}+\gamma\left\|D^{2} \theta\right\|^{2} \\
& \leq\left(\|\eta\|+\left\|\eta_{t}\right\|\right)\|\theta\|-\left(|D u|^{2} D u-\left|D u_{h}\right|^{2} D u_{h}, D \theta\right)+\left\|D^{2} \theta\right\|\|\theta\| \\
& \leq \frac{1}{2}\left(\|\eta\|+\left\|\eta_{t}\right\|\right)^{2}+C\left(\|\theta\|^{2}+\|\eta\|^{2}\right)+\frac{\gamma}{4}\left\|D^{2} \theta\right\|^{2}+\frac{1}{\gamma}\|\theta\|^{2}+\frac{\gamma}{4}\left\|D^{2} \theta\right\|^{2} \\
& \leq\left(C+\frac{1}{2}\right)\left(\|\eta\|+\left\|\eta_{t}\right\|\right)^{2}+\frac{\gamma}{2}\left\|D^{2} \theta\right\|^{2}+\left(C+\frac{1}{\gamma}\right)\|\theta\|^{2} .
\end{aligned}
$$

It then follows from the above inequality that

$$
\frac{d}{d t}\|\theta\|^{2}+\gamma\left\|D^{2} \theta\right\|^{2} \leq(2 C+1)\left(\|\eta\|+\left\|\eta_{t}\right\|\right)^{2}+\left(2 C+\frac{2}{\gamma}\right)\|\theta\|^{2} .
$$

By Gronwall's inequality, we deduce that

$$
\|\theta\|^{2} \leq C^{\prime}\left(\|\theta(0)\|^{2}+\int_{0}^{t}\left(\|\eta(\tau)\|^{2}+\left\|\eta_{t}(\tau)\right\|^{2}\right) d \tau\right) .
$$

Combing (20) and (14) (noticing that $\left.\left(R_{h} u\right)_{t}=R_{h} u_{t}\right)$, and using the triangle inequality, we complete the proof of Theorem 2.2.

Remark 2.3 In Theorem 2.2, we give the $L^{2}$-norm error estimate for the semi-discrete approximation. In fact, we want to obtain some better result for the error estimates. Our best guess on the $H^{2}$-norm error estimate is

$$
\left\|u(t)-u_{h}(t)\right\|_{2} \leq C^{\prime} h^{k-1}\left(\|u(0)\|_{k+1}^{2}+\int_{0}^{T}\left\|u_{t}(\tau)\right\|_{k+1}^{2} d \tau\right)^{\frac{1}{2}}, \quad 0 \leq t \leq T
$$

where the constant $C^{\prime}=C^{\prime}\left(u, u_{h}(0), \gamma\right)$. We will prove it in the next step. 


\section{Full-discrete approximation}

For any given positive integer $M$, let $\Delta t=T / M$ denote the size of the time discretization. Denote $U^{n}=U\left(x, t_{n}\right)$ for $t_{n}=n \Delta t, n=0,1, \ldots, M$. Introduce the forward Euler difference formula,

$$
u_{t}\left(t_{n}\right)=\frac{u^{n}-u^{n-1}}{\Delta t}+\frac{1}{\Delta t} \int_{t_{n-1}}^{t_{n}}\left(\tau-t_{n}\right) u_{t t}(\tau) d \tau=\delta_{t} u^{n}+\varepsilon^{n}
$$

where $u^{n}=u\left(t_{n}\right)$.

Now, we define the full-discrete finite element form to approximate problem (2): Find $U^{n} \in S_{h}^{(k)}(n=1,2, \ldots, M)$ such that

$$
\left\{\begin{array}{l}
\left(\delta_{t} U^{n}, v_{h}\right)+\gamma\left(D^{2} U^{n}, D^{2} v_{h}\right)-\left(D U^{n}, D v_{h}\right)+\left(\left(D U^{n-1}\right)^{2} D U^{n}, D v_{h}\right)=0, \\
\quad \forall v_{h} \in S_{h}^{(k)}, \\
\left(U^{0}-u(0), v_{h}\right)=0, \quad \forall v_{h} \in S_{h}^{(k)} .
\end{array}\right.
$$

For the above form, if $U^{n-1}$ is known and $\Delta t$ sufficiently small, by solving a positive definite system of linear equations which is equal to (22), we can obtain $U^{n}$. Let

$$
u^{n}-U^{n}=u^{n}-R_{h} u^{n}+R_{h} u^{n}-U^{n}=\eta^{n}+\theta^{n}, \quad \theta^{n} \in S_{h}^{(k)} .
$$

Using (2) and (12), $R_{h} u^{n}$ satisfies

$$
\begin{aligned}
& \left(\delta_{t} R_{h} u^{n}, v_{h}\right)+\gamma\left(D^{2} R_{h} u^{n}, D^{2} v_{h}\right) \\
& \quad=\left(\eta^{n}-\varepsilon^{n}-\delta_{t} \eta^{n}, v_{h}\right)+\left(D R_{h} u^{n}, D v_{h}\right)-\left(\left|D u^{n}\right|^{2} D u^{n}, D v_{h}\right) .
\end{aligned}
$$

Adding (22) and (23), $\forall v_{h} \in S_{h}^{(k)}$, we have

$$
\begin{aligned}
& \left(\delta_{t} \theta^{n}, v_{h}\right)+\gamma\left(D^{2} \theta^{n}, D^{2} v_{h}\right) \\
& \quad=\left(\eta^{n}-\varepsilon^{n}-\delta_{t} \eta^{n}, v_{h}\right)+\left(D \theta^{n}, D v_{h}\right)-\left(\left|D u^{n}\right|^{2} D u^{n}-\left|D U^{n-1}\right|^{2} D U^{n}, D v_{h}\right) .
\end{aligned}
$$

Theorem 3.1 Let $u$ be the solution of (2), $U^{n}$ be the solution of (22), $u(0) \in H^{k+1}(I), u_{t} \in$ $L^{2}\left(0, T ; H^{k+1}(I)\right), u_{t t} \in L^{2}\left(0, T ; L^{2}(I)\right), \Delta t / h^{2} \leq c$, and $U^{0} \in S_{h}^{(k)}$ satisfies

$$
\left\|u(0)-U^{0}\right\|_{i} \leq C h^{k+1-i}\|u(0)\|_{2}
$$

where $i=0,1$. Then if $h$ is sufficiently small, there exists a constant $C=C(u, \gamma)$ which is independent of $h, \Delta t$, and $n$, such that

$$
\left\|u^{n}-U^{n}\right\| \leq C\left(\Delta t+h^{k}\right), \quad n=0,1, \ldots, M-1 .
$$

Proof First of all, we give a posterior hypothesis: There exists a $h_{0}$; when $0<h \leq h_{0}$, we have

$$
\left\|D u^{m}-D U^{m}\right\|_{\infty} \leq C, \quad m=1,2, \ldots, n-1
$$

We will prove the correctness of (26) in the end of this proof. 
Lu et al. Boundary Value Problems 2014, 2014:46

Page 8 of 13

http://www.boundaryvalueproblems.com/content/2014/1/46

Setting $v_{h}=\theta^{n}$ in (24), we derive

$$
\begin{aligned}
&\left(\delta_{t} \theta^{n}, \theta^{n}\right)+\gamma\left\|D^{2} \theta^{n}\right\|^{2} \\
& \leq \leq \eta^{n}-\varepsilon^{n}-\delta_{t} \eta^{n}\|\| \theta^{n}\|+\| D \theta^{n}\left\|^{2}-\right\|\left|D u^{n}\right|^{2} D u^{n}-\left|D U^{n-1}\right|^{2} D U^{n}\|\| D \theta^{n} \| \\
& \leq \frac{1}{2}\left\|\eta^{n}-\varepsilon^{n}-\delta_{t} \eta^{n}\right\|^{2}+\frac{1}{2}\left\|\theta^{n}\right\|^{2}+2\left\|D \theta^{n}\right\|^{2} \\
&+\frac{1}{4}\left\|\left|D u^{n}\right|^{2} D u^{n}-\left|D U^{n-1}\right|^{2} D U^{n}\right\|^{2} \\
& \leq \frac{1}{2}\left\|\eta^{n}-\varepsilon^{n}-\delta_{t} \eta^{n}\right\|^{2}+\left(\frac{1}{2}+\frac{2}{\gamma}\right)\left\|\theta^{n}\right\|^{2}+\frac{\gamma}{2}\left\|D^{2} \theta^{n}\right\|^{2} \\
& \quad+\frac{1}{4}\left\|\left|D u^{n}\right|^{2} D u^{n}-\left|D U^{n-1}\right|^{2} D U^{n}\right\|^{2} .
\end{aligned}
$$

Hence

$$
\begin{aligned}
\left\|\theta^{n}\right\|^{2}+\gamma\left\|D^{2} \theta^{n}\right\|^{2} & \\
\leq & \left\|\theta^{n-1}\right\|^{2}+2 \Delta t\left\{\frac{1}{2}\left\|\eta^{n}-\varepsilon^{n}-\delta_{t} \eta^{n}\right\|^{2}+\left(\frac{1}{2}+\frac{2}{\gamma}\right)\left\|\theta^{n}\right\|^{2}\right. \\
& \left.+\frac{1}{4}\left\|\left|D u^{n}\right|^{2} D u^{n}-\left|D U^{n-1}\right|^{2} D U^{n}\right\|^{2}\right\} .
\end{aligned}
$$

Using (14) and (21), we get

$$
\begin{aligned}
\left\|\eta^{n}\right\| & \leq C h^{k+1}\left\|u\left(t_{n}\right)\right\|_{k+1}, \\
\left\|\varepsilon^{n}\right\| & \leq \int_{t_{n-1}}^{t_{n}}\left\|u_{t t}(\tau)\right\| d \tau \leq(\Delta t)^{\frac{1}{2}}\left(\int_{t_{n-1}}^{t_{n}}\left\|u_{t t}(\tau)\right\|^{2} d \tau\right)^{\frac{1}{2}}, \\
\left\|\delta_{t} \eta^{n}\right\| & =\left\|\frac{1}{\Delta t} \int_{t_{n-1}}^{t_{n}} \eta_{t}(\tau) d \tau\right\| \leq C \frac{1}{\Delta t} h^{k+1} \int_{t_{n-1}}^{t_{n}}\left\|u_{t}(\tau)\right\|_{k+1} d \tau \\
& \leq C \frac{1}{(\Delta t)^{\frac{1}{2}}} h^{k+1}\left(\int_{t_{n-1}}^{t_{n}}\left\|u_{t}(\tau)\right\|_{k+1}^{2} d \tau\right)^{\frac{1}{2}} .
\end{aligned}
$$

In addition, we have

$$
\begin{aligned}
\| \mid D u^{n} & \left.\right|^{2} D u^{n}-\left|D U^{n-1}\right|^{2} D U^{n} \| \\
= & \|\left|D u^{n}\right|^{2} D u^{n}-\left|D u^{n-1}\right|^{2} D u^{n}+\left|D u^{n-1}\right|^{2} D u^{n}-\left|D U^{n-1}\right|^{2} D u^{n} \\
& +\left|D u^{n-1}\right|^{2} D u^{n}-\left|D U^{n-1}\right|^{2} D U^{n} \| \\
\leq & \left\|\left|D u^{n}\right|^{2} D u^{n}-\left|D u^{n-1}\right|^{2} D u^{n}\right\|+\left\|\left|D u^{n-1}\right|^{2} D u^{n}-\left|D U^{n-1}\right|^{2} D u^{n}\right\| \\
& +\left\|\left|D U^{n-1}\right|^{2} D u^{n}-\left|D U^{n-1}\right|^{2} D U^{n}\right\| \\
\leq & \left|D u^{n}\right|_{\infty}\left|D u^{n}+D u^{n-1}\right|_{\infty}\left\|D u^{n}-D u^{n-1}\right\| \\
& +\left|D u^{n}\right|_{\infty}^{2}\left|D u^{n-1}+D U^{n-1}\right|_{\infty}\left\|D u^{n-1}-D U^{n-1}\right\| \\
& +\left|D U^{n-1}\right|_{\infty}^{2}\left\|D u^{n}-D U^{n}\right\| .
\end{aligned}
$$


By Theorem 1.1 and Sobolev's embedding theorem, we have

$$
\begin{aligned}
& \left|D u^{n}\right|_{\infty} \leq C\left\|u^{n}\right\|_{2} \leq C \\
& \left|D u^{n}+D u^{n-1}\right|_{\infty} \leq C\left(\left|D u^{n}\right|_{\infty}+\left|D u^{n-1}\right|_{\infty}\right) \leq C\left(\left\|u^{n}\right\|_{2}^{2}+\left\|u^{n-1}\right\|_{2}^{2}\right) \leq C,
\end{aligned}
$$

and

$$
\begin{aligned}
& \left|D U^{n-1}\right|_{\infty} \leq\left|D u^{n-1}-D U^{n-1}\right|_{\infty}+\left|D u^{n-1}\right|_{\infty} \leq C+\left|D u^{n-1}\right|_{\infty} \\
& \left|D u^{n-1}+D U^{n-1}\right|_{\infty} \leq\left|D u^{n-1}-D U^{n-1}\right|_{\infty}+2\left|D u^{n-1}\right|_{\infty} \leq C+\left|D u^{n-1}\right|_{\infty}
\end{aligned}
$$

We have used the posterior hypothesis in (30). Adding (28)-(30) gives

$$
\begin{aligned}
\left\|\left|D u^{n}\right|^{2} D u^{n}-\left|D u^{n-1}\right|^{2} D U^{n}\right\| \\
\leq C\left(\left\|D u^{n}-D u^{n-1}\right\|+\left\|D u^{n-1}-D u^{n-1}\right\|+\left\|D u^{n}-D u^{n}\right\|\right) \\
\leq C\left(\left\|D u^{n}-D u^{n-1}\right\|+\left\|D \theta^{n-1}+D \eta^{n-1}\right\|+\left\|D \theta^{n}+D \eta^{n}\right\|\right) \\
\leq C\left(\int_{t_{n-1}}^{t_{n}}\left\|D u_{t}(\tau)\right\| d \tau+\left\|D \eta^{n-1}\right\|+\left\|D \theta^{n-1}\right\|+\left\|D \eta^{n}\right\|+\left\|D \theta^{n}\right\|\right) \\
\leq C\left[(\Delta t)^{\frac{1}{2}}\left(\int_{t_{n-1}}^{t_{n}}\left\|D u_{t}(\tau)\right\|^{2} d \tau\right)^{\frac{1}{2}}+h^{k}\left(\left\|u\left(t_{n}\right)\right\|_{k+1}+\left\|u\left(t_{n-1}\right)\right\|_{k+1}\right)\right. \\
\left.\quad+\left\|D \theta^{n-1}\right\|+\left\|D \theta^{n}\right\|\right] .
\end{aligned}
$$

Taking the above estimates into (27), we derive

$$
\begin{aligned}
&\left\|\theta^{n}\right\|^{2}-\left\|\theta^{n-1}\right\|^{2}+\gamma\left\|D^{2} \theta^{n}\right\|^{2} \\
& \leq C \Delta t\left(\left\|D \theta^{n}\right\|^{2}+\left\|D \theta^{n-1}\right\|^{2}+h^{2 k}\left\|u\left(t_{n}\right)\right\|_{k+1}^{2}\right) \\
&+C\left[h^{2 k}+(\Delta t)^{2}\right]\left(\int_{t_{n-1}}^{t_{n}}\left(\left\|u_{t}(\tau)\right\|_{k+1}^{2}+\left\|u_{t t}\right\|^{2}\right) d \tau\right) .
\end{aligned}
$$

Taking the sum of $n$, noticing that $\left\|D \theta^{0}\right\| \leq C h^{k}\|u(0)\|_{1}, n \Delta t=t_{n} \leq T$, we obtain

$$
\begin{aligned}
& \left\|\theta^{n}\right\|^{2}+\gamma \sum_{i=1}^{n}\left\|D^{2} \theta^{i}\right\|^{2} \\
& \leq C \Delta t\left[\left\|D \theta^{0}\right\|^{2}+\sum_{i=1}^{n}\left\|D \theta^{i}\right\|^{2}\right] \\
& +C\left[(\Delta t)^{2}+h^{2 k}\right]\left(\int_{0}^{t_{n}}\left(\left\|u_{t}(\tau)\right\|_{k+1}^{2}+\left\|u_{t t}\right\|^{2}\right) d \tau\right) \\
& \leq C \Delta t\left[\left\|D \theta^{0}\right\|^{2}+\frac{1}{2} \sum_{i=1}^{n}\left(\left\|D^{2} \theta^{i}\right\|^{2}+\left\|\theta^{i}\right\|^{2}\right)\right] \\
& +C\left[(\Delta t)^{2}+h^{2 k}\right]\left(\int_{0}^{t_{n}}\left(\left\|u_{t}(\tau)\right\|_{k+1}^{2}+\left\|u_{t t}\right\|^{2}\right) d \tau\right) .
\end{aligned}
$$


Let $\Delta t$ be sufficiently small, which satisfies $\frac{C}{2 \gamma} \Delta t \leq 1$ and $C \Delta t<\frac{1}{2}$, and we deduce

$$
\left\|\theta^{n}\right\|^{2} \leq C \Delta t \sum_{i=1}^{n-1}\left\|\theta^{i}\right\|^{2}+C\left[(\Delta t)^{2}+h^{2 k}\right]
$$

Using the discrete Gronwall inequality, (12), and the triangle inequality, we obtain (25).

Now, in order to complete the proof of Theorem 3.1, we only need to prove the posterior hypothesis (26). Use the inductive method. When $m=0$, based on the initial approximation assumption and the finite element inverse inequality, letting $h \leq h_{0}$ and $h_{0}$ be sufficiently small, we obtain (26). If we assume that (26) is correct for $m=l-1$, based on the above proof, we can easily see that the estimate (25) is correct for $n=l$, where $C$ is a constant independent of $n, \Delta t$, and $h$ (noticing that $t_{n} \leq T$ ). Using the finite element inverse inequality, the interpolation approximation properties, and (25), we have

$$
\begin{aligned}
\left\|D u^{l}-D U^{l}\right\|_{\infty} & \leq C\left\|u^{l}-U^{l}\right\|_{2} \leq C\left(\left\|u^{l}-u_{I}^{l}\right\|_{2}+\left\|u_{I}^{l}-U^{l}\right\|_{2}\right) \\
& \leq C\left(\left\|u^{l}-u_{I}^{l}\right\|_{2}+h^{-2}\left\|u_{I}^{l}-U^{l}\right\|\right) \\
& \leq C\left[\left\|u^{l}-u_{I}^{l}\right\|_{2}+h^{-2}\left(\left\|u_{I}^{l}-u^{l}\right\|+\left\|u^{l}-U^{l}\right\|\right)\right] \\
& \leq C\left(h^{k-2}+h^{-2} \Delta t\right) \leq C
\end{aligned}
$$

where $u_{i} \in S_{h}^{(3)}$ is the Hermite type interpolation approximation of the function $u$. Hence, (26) is correct for $m=l$. Then, using the inductive method, the correctness of (26) is proved, and the proof of Theorem 3.1 is completed.

Remark 3.1 In Theorem 2.2, we give the $L^{2}$-norm error estimate for the full-discrete approximation. In fact, we want to obtain some better result on the error estimates. Our best guess on the $H^{2}$-norm error estimate is

$$
\left\|u^{n}-U^{n}\right\|_{2} \leq C\left(\Delta t+h^{k-2}\right), \quad n=0,1, \ldots, M-1
$$

We will prove it in the next step.

\section{Numerical approximation}

In this section, using the full-discrete form (22), we approximate the solution of problem (1). Let $u_{0}=\cos x, \gamma=1, T=1, h=\frac{\pi}{400}, \Delta t=\frac{1}{100}$. We get the solution which evolves from $t=0$ to $t=1$ (cf. Figure 1$)$.

In addition, we consider the change of error when the time $t=0.5$. Since there is no exact solution to problem (1) to the best of our knowledge, we make a comparison between the solution of (22) on coarse meshes and the fine mesh.

Choose $\Delta t=0.1000,0.0500,0.0250,0.0167,0.0125$, respectively, to solve (22). Set $u_{N}^{\min }(x, 0.5)$ as the solution for $\Delta t_{\min }=0.01$. Denote

$$
\operatorname{err}(0.5, \Delta t)=\left(\int_{0}^{1}\left[u_{N}^{k}(x, 0.5)-u_{N}^{\min }(x, 0.5)\right]^{2} d x\right)^{\frac{1}{2}}, \quad k=1,2,3,4
$$

Then the error is shown in Table 1. 


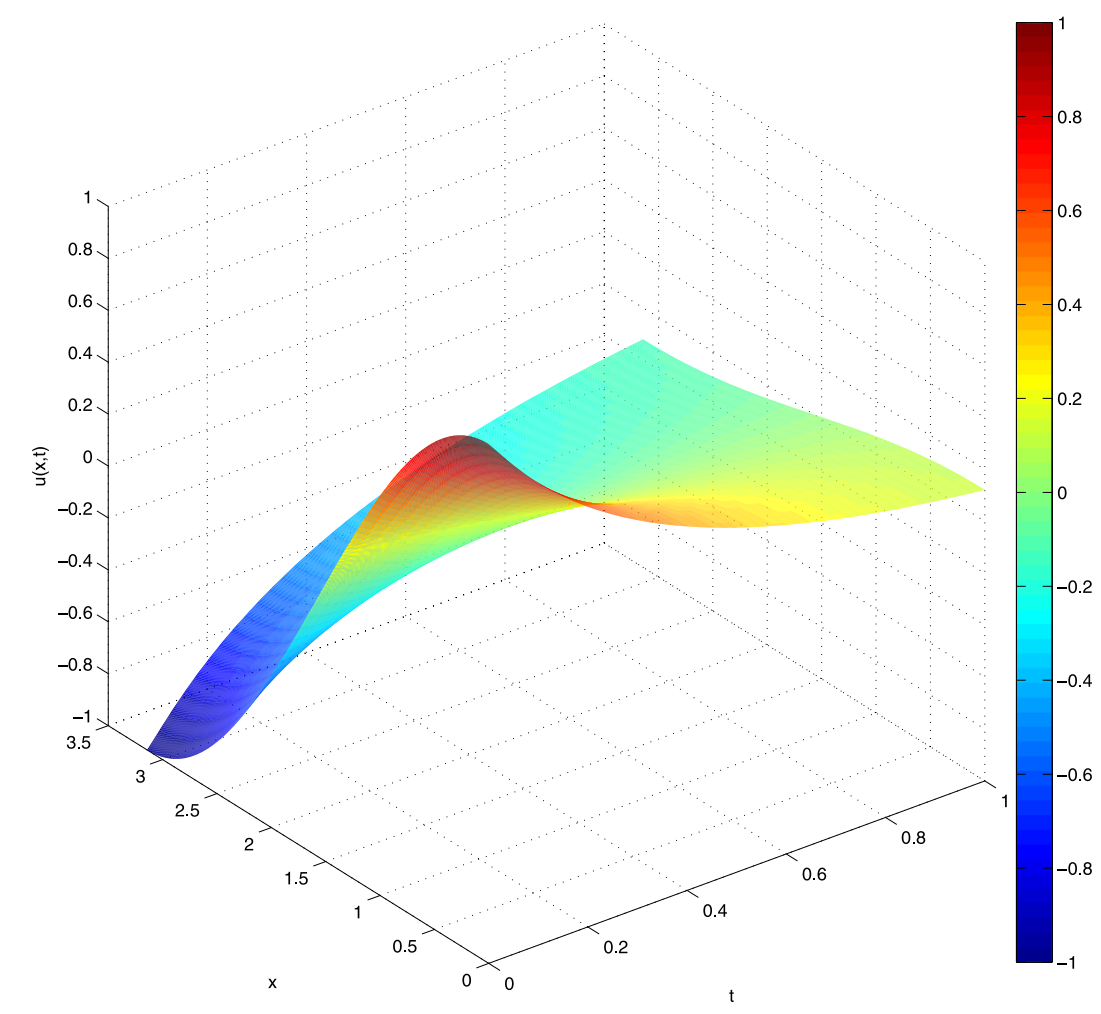

Figure 1 The approximation solution of the full-discrete form.

Table 1 The error for difference time step at $t=0.5$

\begin{tabular}{llcl}
\hline $\boldsymbol{\Delta t}$ & $\operatorname{err}(\mathbf{0 . 5}, \boldsymbol{\Delta t})$ & $\frac{\operatorname{err}(\mathbf{0 . 5}, \boldsymbol{\Delta t})}{\boldsymbol{\Delta t}}$ & $\frac{\operatorname{err}(\mathbf{0 . 5}, \boldsymbol{\Delta} \boldsymbol{t})}{(\boldsymbol{\Delta t})^{\mathbf{2}}}$ \\
\hline 0.1000 & 0.0253 & 0.2530 & 2.5300 \\
0.0500 & 0.0117 & 0.2340 & 4.6800 \\
0.0250 & 0.0045 & 0.1800 & 7.2000 \\
0.0167 & 0.0020 & 0.1198 & 7.1736 \\
0.0125 & $7.5745 e^{-4}$ & 0.0606 & 4.848 \\
\hline
\end{tabular}

In Table 1 , it is easy to see that the third column $\frac{\operatorname{err}(0.5, \Delta t)}{\Delta t}$ is monotone decreasing along with the time step's waning and the fourth column $\frac{\operatorname{err}(0.5, \Delta t)}{(\Delta t)^{2}}$ is not monotone decreasing along with the time step's waning. Then the order of convergence for time is of $O(\Delta t)$ and $O\left((\Delta t)^{2}\right)$. It is easy to see that the result of the numerical analysis on time is better than the theoretical result. The reason may be the existence of a nonlinear term or the limit of the theoretical proof tool.

Now, we consider the error for the difference $h$ at $t=0.5$. We choose $h=0.1517,0.0785$, $0.0628,0.0524$, respectively, to solve $(22)$. Set $u_{N}^{\min }(x, 0.5)$ as the solution for $h=\frac{\pi}{400}, \Delta t=$ $\frac{1}{100}$, and use the discrete $L^{2}$ norm to obtain the error $\operatorname{err}(0.5, h)$ for $t=0.5$. Then the error is shown in Table 2.

In Table 2 , it is easy to see that the fourth column $\frac{\operatorname{err}(0.5, h)}{h^{2}}$ is monotone decreasing along with the space step's waning. The fifth column $\frac{\operatorname{err}(0.5, h)}{h^{3}}$ is not monotone increasing along with the space step's waning, and it tends to a positive constant when the space subdivision 
Table 2 The error for difference $h$ at $t=0.5$

\begin{tabular}{lllll}
\hline $\boldsymbol{h}$ & $\operatorname{err}(\mathbf{0 . 5}, \boldsymbol{h})$ & $\frac{\operatorname{err}(\mathbf{0 . 5}, \boldsymbol{h})}{\boldsymbol{h}}$ & $\frac{\operatorname{err}(\mathbf{0 . 5}, \boldsymbol{h})}{\boldsymbol{h}^{\mathbf{2}}}$ & $\frac{\operatorname{err}(\mathbf{0 . 5}, \boldsymbol{h})}{\boldsymbol{h}^{\mathbf{3}}}$ \\
\hline 0.1571 & $2.6459 e^{-7}$ & $1.6842 e^{-6}$ & $1.0721 e^{-5}$ & $6.8241 e^{-5}$ \\
0.1047 & $3.6393 e^{-8}$ & $3.4759 e^{-7}$ & $3.3199 e^{-6}$ & $3.1709 e^{-5}$ \\
0.0785 & $1.8007 e^{-8}$ & $2.2939 e^{-7}$ & $2.9222 e^{-6}$ & $3.7225 e^{-5}$ \\
0.0628 & $4.9313 e^{-9}$ & $7.8524 e^{-8}$ & $1.2504 e^{-6}$ & $1.9911 e^{-5}$ \\
0.0524 & $9.5773 e^{-10}$ & $1.8277 e^{-8}$ & $3.4880 e^{-7}$ & $6.6566 e^{-6}$ \\
\hline
\end{tabular}

Table 3 The error for the differences $h$ and $\Delta t$ at $t=0.5$

\begin{tabular}{llll}
\hline $\boldsymbol{h}$ & $\boldsymbol{\Delta t}$ & $\operatorname{err}(\mathbf{0 . 5}, \boldsymbol{h}, \boldsymbol{\Delta t})$ & $\frac{\operatorname{err}(\mathbf{0 . 5}, \boldsymbol{h}, \boldsymbol{\Delta t})}{\boldsymbol{h}^{\mathbf{3}} \mathbf{\Delta} \boldsymbol{t}}$ \\
\hline 0.3927 & 0.1000 & 0.0525 & 0.3270 \\
0.2614 & 0.0500 & 0.0266 & 0.3286 \\
0.1963 & 0.0250 & 0.0125 & 0.3839 \\
0.1571 & 0.0167 & 0.0062 & 0.3018 \\
0.1257 & 0.0125 & 0.0026 & 0.1795 \\
\hline
\end{tabular}

is small enough. Hence, we can find a positive constant $C$, such that

$$
\frac{\operatorname{err}(0.5, h)}{h^{3}} \leq \mathcal{C}
$$

which means the order of the error estimates is $O(h)^{3}$.

On the other hand, we consider the error for difference $h$ and $\Delta t$ at $t=0.5$. We choose $(h, \Delta t)=\left(\frac{\pi}{8}, \frac{1}{10}\right),\left(\frac{\pi}{10}, \frac{1}{20}\right),\left(\frac{\pi}{16}, \frac{1}{40}\right),\left(\frac{\pi}{20}, \frac{1}{60}\right),\left(\frac{\pi}{25}, \frac{1}{80}\right)$, respectively, to solve (22), set $u_{N}^{\min }(x, 0.5)$ as the solution for $h=\frac{\pi}{400}, \Delta t=\frac{1}{100}$, use the discrete $L^{2}$ norm to obtain the error $\operatorname{err}(0.5, h, \Delta t)$ for $t=0.5$, which is shown in Table 3 .

In Table 3, it is easy to see that the fourth column $\frac{\operatorname{err}(0.5, h, \Delta t)}{h^{3}+\Delta t}$ tends to a positive constant. Hence, we can find a positive constant $C$, such that

$$
\frac{\operatorname{err}(0.5, h, \Delta t)}{h^{3}+\Delta t} \leq \mathcal{C}
$$

which means that the order of error estimates is of $O\left(h^{3}+\Delta t\right)$.

\section{Competing interests}

The authors declare that they have no competing interests.

Authors' contributions

$X Z$ and FL wrote the first draft, FL made the figure of numerical solution and results on errors, BL and XZ corrected and improved the final version. All authors read and approved the final draft.

\section{Author details}

${ }^{1}$ College of Mathematics, Jilin University, Changchun, 130012, China. ${ }^{2}$ School of Science, Jiangnan University, Wuxi, 214122, China.

\section{Acknowledgements}

The authors would like to express their deep thanks for the referee's valuable suggestions about the revision and improvement of the manuscript.

Received: 11 October 2013 Accepted: 7 February 2014 Published: 24 Feb 2014

\section{References}

1. Barrett, JW, Blowey, JF, Garcke, H: Finite element approximation of the Cahn-Hilliard equation with degenerate mobility. SIAM J. Numer. Anal. 37, 286-318 (1999)

2. Choo, SM, Kim, YH: Finite element scheme for the viscous Cahn-Hilliard equation with a nonconstant gradient energy coefficient. J. Appl. Math. Comput. 19, 385-395 (2005) 
3. Elliott, CM, French, DA: A nonconforming finite element method for the two-dimensional Cahn-Hilliard equation. SIAM J. Numer. Anal. 26, 884-903 (1989)

4. Feng, $\mathrm{X}, \mathrm{Wu}, \mathrm{H}$ : A posteriori error estimates for finite element approximations of the Cahn-Hilliard equation and the Hele-Shaw flow. J. Comput. Math. 26, 767-796 (2008)

5. Kovács, M, Larsson, S, Mesforush, A: Finite element approximation of the Cahn-Hilliard-Cook equation. SIAM J. Numer. Anal. 49, 2407-2429 (2011)

6. Zhang, T: Finite element analysis for Cahn-Hilliard equation. Math. Numer. Sin. 28, 281-292 (2006)

7. King, BB, Stein, O, Winkler, M: A fourth order parabolic equation modeling epitaxial thin film growth. J. Math. Anal. Appl. 286, 459-490 (2003)

8. Zangwill, A: Some causes and a consequence of epitaxial roughening. J. Cryst. Growth 163, 8-21 (1996)

9. Kohn, RV, Yan, X: Upper bound on the coarsening rate for an epitaxial growth model. Commun. Pure Appl. Math. 56 1549-1564 (2003)

10. Liu, C: Regularity of solutions for a fourth order parabolic equation. Bull. Belg. Math. Soc. Simon Stevin 13(3), 527-535 (2006)

11. Zhao, X, Liu, C: The existence of global attractor for a fourth-order parabolic equation. Appl. Anal. 92, 44-59 (2013)

12. Zhao, X, Liu, C: Optimal control of a fourth-order parabolic equation modeling epitaxial thin-film growth. Bull. Belg. Math. Soc. Simon Stevin 20(3), 547-557 (2013)

13. Ciarlet, P: The Finite Element Method for Elliptic Problems. North-Holland, Amsterdam (1978)

10.1186/1687-2770-2014-46

Cite this article as: Liu et al.: Finite element analysis of a nonlinear parabolic equation modeling epitaxial thin-film growth. Boundary Value Problems 2014, 2014:46

\section{Submit your manuscript to a SpringerOpen ${ }^{\ominus}$ journal and benefit from:}

- Convenient online submission

- Rigorous peer review

- Immediate publication on acceptance

- Open access: articles freely available online

- High visibility within the field

- Retaining the copyright to your article 